\title{
Patients Who Avoid Wheat and Gluten: Is That Health or Lifestyle?
}

\author{
Imran Aziz • David S. Sanders
}

Published online: 5 March 2014

(c) Springer Science+Business Media New York 2014

In a New York restaurant, a celiac patient requesting a gluten-free menu was asked by the server if the diet was for health or for lifestyle. This increasingly common exchange exemplifies the clinical conundrum clinicians face regarding the use of a gluten-free diet (GFD).

Although humankind has likely existed in some form for 2.5 million years, it is only in the last 10,000 years that humans have been exposed to wheat, which was originally cultivated in the Fertile Crescent from $\sim 9000$ BC to 4000 $\mathrm{BC}$. Thus, wheat and therefore, gluten is a relatively novel component of the human diet. The globalization of wheat production, and subsequently its increased worldwide consumption, has increased the incidence and clinical recognition of gluten-related disorders [1].

The most notable gluten-related disorder is celiac disease (CD), a state of heightened immune response to ingested gluten in genetically susceptible individuals. Initial reports in the 1950s suggested this to be a rare condition affecting 1 in 8,000. Nevertheless, more recently, its prevalence in the United States and elsewhere is $0.71 \%$ or 1 in 141 [2]. The sole effective treatment for CD is lifelong adherence to a strict GFD.

More recently, a few case reports and series published in the 1980s described individuals intolerant to gluten, by way of experiencing irritable bowel syndrome (IBS) type symptoms, but in whom CD serologies and small intestinal biopsies were normal. Since the diagnosis or explanation for these symptoms was lacking and possibly dismissed, such patients were unclassifiable and untreatable [3]. Nonetheless, over the last few years, mounting media

I. Aziz $(\bowtie) \cdot$ D. S. Sanders

Department of Gastroenterology, Room P39, Royal Hallamshire Hospital, Sheffield Teaching Hospitals, Sheffield S10 2JF, UK e-mail: imran.aziz@sth.nhs.uk reports have speculated that the use of a GFD is rapidly growing to the point that it is now a big business, seemingly greatly exceeding the demand predicted from $\mathrm{CD}$ subjects alone. This media pressure finally prodded the scientific community into a response, led by an Australian group, who performed a landmark double-blind, randomised, placebo-controlled study reporting that gluten from two slices of bread and one muffin indeed induced gastrointestinal symptoms in subjects without CD [4]. Subsequently, a consensus of experts has introduced the term "non-celiac gluten sensitivity" (NCGS) to describe such subjects [5]. A subsequent flurry of research papers raised the ratio of publications for NCGS:CD from 1:438 to 1:10, to the point that NCGS is considered the new frontier of gluten-related disorders [6].

The pathophysiology of NCGS has not yet been fully elucidated. Whereas the innate and adaptive immune response to gluten is responsible for the manifestations seen in $\mathrm{CD}$, only the innate immune system is seemingly triggered in NCGS. There have been promising reports of altered small bowel intestinal permeability, proliferation of peripheral blood monocytes, the presence of antigliadin antibodies, enhanced cytokine induction, and induction of basophil activation in NCGS, although these findings have not yet been confirmed [6]. Gluten is only one of the complex milieu of nutrients present in wheat, which also contains fructans, which are fermentable, poorly absorbed, short chain carbohydrates, also termed FODMAPs (fermentable oligosaccharides, disaccharides, monosaccharides and polyols). FODMAPS provoke gastrointestinal symptoms in IBS patients through mechanisms involving gut microbiota, gas production, and fermentation [7]. There is also emerging evidence that wheat-amylase trypsin inhibitors are also associated with intestinal symptoms [8]. Since it is unclear which component of wheat-based products is 
responsible for an individual's symptoms, it may be premature to assign all of the blame to gluten. In a recent study, the same team from Australia reported that reduction of dietary FODMAPs in patients with self-perceived NCGS was the major contributing factor to symptom relief [9]. Subjects who were already well controlled on a GFD reaped further benefit from the reduction of dietary FODMAPs. In contrast, a double-blind, randomized, placebo-controlled crossover study of low and high-dose gluten and whey protein containing diets failed to demonstrate specific or dose-dependent effects of dietary gluten, contrary to results previously reported by the same team. Although such conflicting studies challenge the concept of NCGS, the investigators nonetheless concluded that the NCGS is a true entity, with differences between the two studies attributed to variations of the glutens used or the hypothesis a moderate content of FODMAPS are necessary for gluteninduced symptoms. In light of these data, the classification of NCGS may be overly restrictive; terms such as "nonceliac wheat sensitivity" (NCWS) or "patients who avoid wheat and gluten" (PWAWG) although somewhat awkward, may be more accurate albeit more confusing [10,11].

With regard to the prevalence of gluten or wheat avoidance, the number of individuals embracing a GFD far exceeds the number diagnosed with $\mathrm{CD}$. The US National Health and Nutrition Examination Survey (NHANES), involving 7,798 people aged 6 years or older, reported that $0.63 \%$ of the American public consumes a GFD although the majority of these do not have CD [2]. In New Zealand, CD affects $1 \%$ of children, yet $5 \%$ report gluten avoidance [12]. Most recently, we have reported that in a large population $(n=1,002)$ living in the United Kingdom, sensitivity to gluten-based products is self-reported by $13 \%$ of the population, with $3.7 \%$ consuming a GFD, even though only $0.8 \%$ are aware that they have been formally diagnosed with CD [13]. Furthermore, gluten-sensitive individuals referred to secondary care are predominantly female, report an association with IBS, and experience intestinal and extraintestinal symptoms after gluten ingestion. Moreover, patients in secondary care with self-reported gluten sensitivity were similar to individuals reporting gluten sensitivity within the population cohort [13].

In this Issue of Digestive Disease and Sciences, Tavakkoli et al. [11] report on the characteristics of patients diagnosed with PWAWG. They report that 84 PWAWG and $585 \mathrm{CD}$ patients shared some similarities, such as autoimmune comorbidities, mean body mass index (BMI) and mean haemoglobin value. However, individuals with PWAWG had lower mean BMI, haemoglobin and blood pressure when compared to 2,686 NHANES subjects. Although some of the findings may be due to the effects of a GFD, the investigators also speculate that the shared immunologic pathways between $\mathrm{CD}$ and PWAWG may have a beneficial role on blood pressure; $60 \%$ of PWAWG were positive for the human leukocyte antigen typing for DQ2 or DQ8, whereas the predicted prevalence of HLA-DQ in the general population is $\sim 25 \%$. Thirty percent of the PWAWG subjects carried alternate diagnoses such as small bowel bacterial overgrowth or other food intolerances. Although novel, the study is limited by its small sample size, retrospective nature, and non-standardized protocol.

In summary, there is a growing percentage of individuals who describe intolerances to wheat-based products in the absence of CD. Since the wheat component responsible for symptom production is currently unknown it may be argued that these patients should alternately be diagnosed as having NCWS or PWAWG. Regardless of designations, wheat-sensitive individuals have a higher prevalence of positive HLA-DQ typing than in the general population. Furthermore, many of these individuals may have excessive fermentation from small intestinal bacterial overgrowth, which could either be co-incidental or actually responsible for the complaints of PWAWG. The investigators should be congratulated for providing a clinical phenotype for PWAWG that at the least will provide data that will enhance patient counselling. Future challenges will include identifying the culprit wheat component and elucidating host-microbiome interactions in this condition.

\section{Key Messages}

- The globalization of wheat production and consumption has unmasked the presence of gluten-related disorders.

- Celiac disease (CD) affects $0.71 \%$ of the United States population, yet gluten-free diets are of increasing popularity in non-CD subjects.

- Gluten induces gastrointestinal symptoms in patients without celiac disease, termed non-celiac gluten sensitivity (NCGS).

- As wheat based products contain many components which can also induce intestinal symptoms, the terms "non-celiac wheat sensitivity" (NCWS) or "patients who avoid wheat and gluten" (PWAWG) have been suggested.

- Shared immunologic pathways may explain some of the similarities seen in PWAWG and CD.

Conflict of interest None.

\section{References}

1. Aziz I, Sanders DS. Emerging concepts: from coeliac disease to non-coeliac gluten sensitivity. Proc Nutr Soc. 2012;71:576-580. 
2. Rubio-Tapia A, Ludvigsson JF, Brantner TL, Murray JA, Everhart JE. The prevalence of celiac disease in the United States. Am J Gastroenterol. 2012;107:1538-1544 (quiz 1537, 1545).

3. Verdu EF, Armstrong D, Murray JA. Between celiac disease and irritable bowel syndrome: the "no man's land" of gluten sensitivity. Am J Gastroenterol. 2009;104:1587-1594.

4. Biesiekierski JR, Newnham ED, Irving PM, et al. Gluten causes gastrointestinal symptoms in subjects without celiac disease: a double-blind randomized placebo-controlled trial. Am J Gastroenterol. 2011;106:508-514 (quiz 515).

5. Sapone A, Bai JC, Ciacci C, et al. Spectrum of gluten-related disorders: consensus on new nomenclature and classification. BMC Med. 2012;10:13.

6. Catassi C, Bai JC, Bonaz B, et al. Non-Celiac Gluten sensitivity: the new frontier of gluten related disorders. Nutrients. 2013;5:3839-3853.

7. Halmos EP, Power VA, Shepherd SJ, Gibson PR, Muir JG. A diet low in FODMAPs reduces symptoms of irritable bowel syndrome. Gastroenterology 2014;146:67.e5-75.e5.

8. Tilg H, Koch R, Moschen AR. Proinflammatory wheat attacks on the intestine: alpha-amylase trypsin inhibitors as new players. Gastroenterology 2013;144:1561-1563 (discussion 1563-1564).
9. Biesiekierski JR, Peters SL, Newnham ED, Rosella O, Muir JG, Gibson PR. No effects of gluten in patients with self-reported non-celiac gluten sensitivity after dietary reduction of fermentable, poorly absorbed, short-chain carbohydrates. Gastroenterology 2013;145:320-328.e1-3.

10. Carroccio A, Rini G, Mansueto P. Non-celiac wheat sensitivity is a more appropriate label than non-celiac gluten sensitivity. Gastroenterology. 2014;146:320-321.

11. Tavakkoli A, Lewis S, Tennyson C, Lebwohol B, Green P. Characteristics of patients who avoid wheat and/or gluten in the absence of celiac disease. Dig Dis Sci. (Epub ahead of print). doi:10.1007/s10620-013-2981-6.

12. Tanpowpong $P$, Ingham TR, Lampshire $P K$, et al. Coeliac disease and gluten avoidance in New Zealand children. Arch Dis Child. 2012;97:12-16.

13. Aziz I, Lewis NR, Hadjivassiliou M, et al. A UK study assessing the population prevalence of self-reported gluten sensitivity and referral characteristics to secondary care. Eur J Gastroenterol Hepatol. 2014;26:33-39. 